\title{
A Dynamical Control View on Synchronization
}

\author{
Reportnr. 2000.04
}

H. Nijmeijer

\section{Address}

University of Twente,

Faculty of Mathematical Sciences

P.O. Box 217,

7500 AE Enschede,

The Netherlands

Eindhoven University of Technology

Faculty of Mechanical Engineering

P.O. Box 513,

5600 MB Eindhoven

The Netherlands 


\title{
A Dynamical Control View on Synchronization
}

\author{
H. Nijmeijer \\ University of Twente, Faculty of Mathematical Sciences \\ P.O.Box 217, 7500 AE Enschede, The Netherlands \\ Eindhoven University of Technology, Faculty of Mechanical Engineering \\ P.O.Box 513, 5600 MB Eindhoven, The Netherlands
}

\begin{abstract}
Synchronization of complex/chaotic systems is reviewed from a dynamical control perspective. It is shown that notions like observer and feedback control are essential in the problem of how to achieve synchronization between two systems on the basis of partial state measurements of one of the systems. Examples are given to demonstrate the main results.
\end{abstract}

Keywords: synchronization, dynamics, chaos control, uncertainty

\section{Introduction}

Probably one of the earliest detailed accounts on synchronized motion was made by Christiaan Huygens, who around 1650 describes in his notebook (Huijgens 1673) an experiment where two identical pendulum clocks are attached to the same (flexible) bar, and these clocks exhibit synchronized motion in a short while in case they are initialized at different phases. The explanation by Huijgens is remarkably accurate since by that time the differential calculus needed to describe the clocks' motion was still to be developed. Many other examples of synchronized motion have been described after the 17th century. For instance, Rayleigh describes in his famous treatise "The theory of sound" (Rayleigh 1945) in 1877 that two organ tubes may produce a synchronized sound provided the outlets are close to each other. Eearly this century another Dutch scientist, B. van der Pol, studied synchronization of certain (electrical-) mechanical systems, see (van der Pol 1920). Actually, rotating bodies, or more general rotating mechanical structures form a very important and special class of systems that, with or without the interaction through some coupling, exhibit synchronized motion. In.fact, synchronization of oscillating physical systems is by today an important subject in some of the major physics journals. An illuminating survey on synchronization of a wide variety of mostly (electrical-) mechanical systems is given in (Blekhman et al. 1995) Also (Parlitz and Kocarev 1999) contains a rich class of motivating and illustrative examples of synchronizing systems. The growing interest in synchronization - and the above mentioned surveys are illustrative for this - was probably caused by the paper (Pecora and Carroll 1990) where, among others, secure communication as a potential application has been indicated. Although sofar it is still questionable whether this application can be fully realized, the Pecora and Carroll paper has formed an impulse for much research along these lines.

On the other hand, for mechanical systems synchronization is of utmost importance as soon as two machines have to cooperate. Typically robot coordination, cf. (Brunt 1998) and cooperation of manipulators, see (Liu et al. 1999) form important illustrations of the same goal, where it is desired that two or more mechanical systems, either identical or different, are asked to work in synchrony.

The purpose of this paper is to address the synchronization problem from a control theory perspective. Control theory is the field in which a systematic study of control systems, together with appropriate controller design(s) is made, see e.g. (Kailath 1980) as a textbook on linear 
control theory and (Nijmeijer and van der Schaft 1990) for results in nonlinear control. Control theory, and more specific nonlinear control, forms a powerful framework to formulate and describe various synchronization questions, and the aim of the paper is to present a review of some of the existing tools and methods from nonlinear control in this regard. This includes the obvious problem of how to build a synchronization system for a given system; a problem that is intimately linked with an observer problem in control, see section 2. In section 3 various aspects regarding (parameter) uncertainty and noise are reviewed. Especially in practical applications like communications and coordination these noise and robustness issues are of great importance. Controlled synchronization is the subject of section 4 . Contrary to the setting of section 2, where for a given system a synchronizing system is sought, here the problem is to achieve synchronization of two system by means of a suitably feedback controller.

The paper does not present formal theorems and proofs but merely develops various problemsolutions through illustrative examples. For detailed formulations and proofs the reader has to consult the appropriate references. Hopefully, the paper initiates further interest in dynamical control methods in the study of synchronization problems.

Acknowledgements Lot of the research reported here has been done in cooperation with colleagues and friends, who as co-authors appear in the references at the end. Their help in this research is warmly acknowledged.

\section{Synchronization and observers}

Following (Pecora and Carroll 1990) we consider the Lorenz system

$$
\left\{\begin{array}{l}
\dot{x}_{1}=\sigma\left(y_{1}-x_{1}\right) \\
\dot{y}_{1}=r x_{1}-y_{1}-x_{1} z_{1} \\
\dot{z}_{1}=-b z_{1}+x_{1} y_{1}
\end{array}\right.
$$

The system (1) is known to exhibit complex or chaotic motions for certain parameters $\sigma, r, b>0$. With the system (1) viewed as the transmitter, or master system, we introduce the drive signal

$$
y=x_{1}
$$

which can be used at the receiver, or slave system, to achieve asymptotic synchronization. This means, as in (Pecora and Carroll 1990), we take as receiver dynamics

$$
\left\{\begin{array}{l}
\dot{x}_{2}=\sigma\left(y_{2}-x_{2}\right) \\
\dot{y}_{2}=r x_{1}-y_{2}-x_{1} z_{2} \\
\dot{z}_{2}=-b z_{2}+x_{1} y_{2}
\end{array}\right.
$$

Notice that (3) consists of a copy of (1), with state $\left(x_{2}, y_{2}, z_{2}\right)$ and where in the $\left(y_{2}, z_{2}\right)$-dynamics the known signal $x_{1}$, see (2), is substituted for $x_{2}$. Introducing the error variables $e_{1}=x_{1}-x_{2}$, $e_{2}=y_{1}-y_{2}, e_{3}=z_{1}-z_{2}$ we obtain the error dynamics

$$
\left\{\begin{array}{l}
\dot{e}_{1}=\sigma\left(e_{2}-e_{1}\right) \\
\dot{e}_{2}=-e_{2}-x_{1} e_{3} \\
\dot{e}_{3}=-b e_{3}+x_{1} e_{2}
\end{array}\right.
$$

which is a linear time-varying system. The stability of $\left(e_{1}, e_{2}, e_{3}\right)=(0,0,0)$ is straightforwardly checked using the Lyapunov-function

$$
V\left(e_{1}, e_{2}, e_{3}\right)=\frac{1}{\sigma} e_{1}^{2}+e_{2}^{2}+e_{3}^{2}
$$

with time-derivative along (4)

$$
\dot{V}\left(e_{1}, e_{2}, e_{3}\right)=-2\left(e_{1}-\frac{1}{2} e_{2}\right)^{2}-\frac{3}{2} e_{2}^{2}-2 b e_{3}^{2}
$$


showing that $\left(e_{1}, e_{2}, e_{3}\right)$ asymptotically (and even exponentially!) converges to $(0,0,0)$. In other words, the receiver dynamics (3) asymptotically synchronizes with the chaotic transmitter (1) no matter how (1) and (3) are initialized.

Corollary 1 Almost similarly, one can show that the $\left(y_{2}, z_{2}\right)$ dynamics from (3) - which are independent from $x_{2}$ anyways - will synchronize with $\left(y_{1}, z_{1}\right)$ from (1), using the Lyapunov function $V\left(e_{2}, e_{3}\right)=e_{2}^{2}+e_{3}^{2}$. This also implies that in this manner the state $\left(x_{1}, y_{1}, z_{1}\right)$ can be reconstructed from $\left(y_{2}, z_{2}\right)$ and the known signal $x_{1}$.

The synchronization of the transmitter (1) and receiver (3) using the drive signal (2) may at this point seem more a coincidence rather than a structural property. However, as will be argued, this is not the case, but follows in much more generality. In particular one may cast the foregoing into an observer problem. To that end consider the general system

$$
\dot{x}=f(x)
$$

with $x \in \mathbb{R}^{n}, f$ a smooth vectorfield, and output (or measurement)

$$
y=h(x)
$$

for some smooth function $h$. Note that more generally one may consider $(7,8)$ on a manifold with function $h$ mapping into another manifold. The observer problem can be formulated as: Given $y(t), t \geq 0$, reconstruct asymptotically $x(t), t \geq 0$.

Corollary 2 In the same style the reduced observer problem reads as the question of reconstructing $x(t)(\bmod y(t))$, given $y(t), t \geq 0$. As an easy example, consider the linear system

$$
\left\{\begin{array}{l}
\dot{x}_{1}=y_{1} \\
\dot{y}_{1}=a x_{1}+b y_{1}
\end{array}\right.
$$

with output

$$
y=x_{1}
$$

Setting, for some $k \in \mathbb{R}$,

$$
z=y_{1}+k x_{1}
$$

we see that

$$
\dot{z}=(b+k) z+\left(a-b k-k^{2}\right) y
$$

ans thus we may find a reduced observer as

$$
\dot{\tilde{z}}=(b+k) \tilde{z}+\left(a-b k-k^{2}\right) y
$$

provided $b+k<0$. The state $\left(x_{1}, y_{1}\right)$ is asymptotically reconstructed as $(y, \tilde{z}-k y)$.

With the given formulation of the observer problem at hand the natural question is, how to find, given $(7,8)$, a mechanism for reconstructing $x(t), t \geq 0$. Although in its full generality the answer to the above question is unknown, there are some important cases where a solution can be found. Some of them will be reviewed next. The natural way to approach the observer problem for ( 7 , 8 ) is to design another dynamical system driven by the measurements (8)

$$
\dot{\tilde{x}}=f(\tilde{x})+k(\tilde{x}, y)
$$

where the $y$-parametrized vectorfield $k$ in (14) should be such that $k(\tilde{x}, y)=0$ if $h(\tilde{x})=h(x)=y$. The dynamics (14) is called an observer for (7) provided that $\tilde{x}(t)$ asymptotically converges to $x(t)$, for any pair of initial conditions $x(0)$ and $\tilde{x}(0)$. The structure of the observer (14) deserves some further attention. One may view (14) as an identical copy of (7) with an 'innovations' term $k(\tilde{x}, y)$ which vanishes in case the estimated output $\tilde{y}=h(\tilde{x})$ coincides with $y=h(x)$. The latter 
could be phrased as we can not do better as our measurements allow for. In the Lorenz system $(1,2)$ with receiver (3), it is easily checked that the system (3) indeed acts as an observer and can be put in the form (14):

$$
\begin{cases}\dot{x}_{2}=\sigma\left(y_{2}-x_{2}\right) & +0 \\ \dot{y}_{2}=r x_{2}-y_{2}-x_{2} z_{2} & +\left(r-z_{2}\right)\left(x_{1}-x_{2}\right) \\ \dot{z}_{2}=-b z_{2}+x_{2} y_{2} & +y_{2}\left(x_{1}-x_{2}\right)\end{cases}
$$

Also, it may be worth noting that (14) is simply a computerized model and no hard-ware is required in building this system, even if a hardware realization of (7) is given.

Corollary 3 (i) Though we restrict attention to observers of the form (14) with dynamics of the same dimension as (7), other possibilities for obtaining suitable estimates for $x(t)$ exist. For instance, the estimate $\tilde{x}(t)$ can arise as a function of a higher dimensional measurement driven dynamics, or even as a solution of an infinite dimensional (pde) system.

(ii) It should be clear, see also Corollary 2, that a reduced order observer should be designed as a measurement driven dynamics that asymptotically matches with $x(t)(\bmod y(t))$. Although some interesting aspects arise, no further attention to reduced observers will be given here.

To illustrate the above observer design, we discuss first the linear observer problem, (Kailath 1980 ); that is both the dynamics (7) and measurements (8) are assumed to be linear:

$$
\begin{aligned}
& \dot{x}=A x \\
& y=C x
\end{aligned}
$$

with $x \in \mathbb{R}^{n}, y \in \mathbb{R}$ and $A$ and $C$ matrices of corresponding dimensions. An observer in this case should be of the form

$$
\dot{\tilde{x}}=A \tilde{x}+K(C x-C \tilde{x})
$$

which, setting $e=x-\tilde{x}$, yields the error dynamics

$$
\dot{e}=(A-K C) e
$$

Clearly, (18) acts as an observer for (16), or what is the same, $\tilde{x}$ and $x$ asymptotically synchronize, if (19) has $e=0$ as asymptotically stable equilibrium. The question under what conditions a matrix $K$ can be found so that (17) is asymptotically stable can be answered using the observability rank condition. The linear system $(16,17)$ satisfies the observability rank condition if

$$
\operatorname{rank}\left[\begin{array}{c}
C \\
C A \\
\vdots \\
C A^{n-1}
\end{array}\right]=n
$$

which is equivalent to the requirement that the system $(16,17)$ is observable, i.e., the state $x(t)$ is uniquely determined by $y(t), t \geq 0$. The rank condition (20) is equivalent to the pole placement property which means that for any symmetric set of $n$ points in $\mathbb{C}$, there exists a real matrix $K$ such that $A-K C$ has these $n$ points as eigenvalues. In particular, it follows that (20) guarantees the existence of an observer (18) (or suitable $K$ ) that makes (19) asymptotically stable. In fact a slightly weaker condition than (20), detectability, is required for the stabilizability of (19) with a suitable $K$. Detectability requires, instead of (20), that $A$ restricted to the largest $A$-invariant subspace in $\operatorname{Ker} C$, should be asymptotically stable. For further details, see (Kailath 1980).

It is clear that the above discussion on synchronization of linear systems can not directly be used for nonlinear/chaotic systems. On the other hand, there are a number of extensions of the 
foregoing linear observer design that are relevant for complex nonlinear systems. The first class for which observer design is as simple as in the linear case are the so-called Lur'e systems, which are described as

$$
\begin{gathered}
\dot{x}=A x+\varphi(C x) \\
y=C x
\end{gathered}
$$

with the pair $(A, C)$ observable, i.e. (20) holds, and $\varphi$ is a smooth nonlinear vector field depending on $y$. A synchronizing system (observer) is designed as

$$
\dot{\bar{x}}=A \tilde{x}+\varphi(C x)+K(C x-C \tilde{x})
$$

which again produces the error dynamics (19). Notice that the class of systems $(21,22)$ only contain nonlinearities in the dynamics that depend upon the measured output $y$, and which thus also can be used in the observer (23). Perhaps the best known example of the form $(21,22)$ is the Chua circuit:

$$
\left\{\begin{array}{l}
\dot{x}_{1}=\alpha\left(-x_{1}+y_{1}-\varphi\left(x_{1}\right)\right) \\
\dot{y}_{1}=x_{1}-y_{1}+z_{1} \\
\dot{z}_{1}=-\lambda y_{1}
\end{array}\right.
$$

where $\varphi\left(x_{1}\right)=m_{1} x_{1}+m_{2}\left(\left|x_{1}+1\right|-\left|x_{1}-1\right|\right)$, with $m_{1}=-\frac{5}{7}, m_{2}=-\frac{3}{7}$, and $23<\lambda<31, \alpha=$ 15.6. Taking as measurements

$$
y=x_{1}
$$

one immediately realizes that this system - which is chaotic and has a so-called double scroll attractor - is of Lur'e type and admits an observer of the form (23), since the corresponding linear part is observable. It is interesting to see that the only nonlinearity in (24) is through the piecewise linear function (non-smooth) $\varphi$, which only depends on the measurements $y$.

A larger class of systems that admit linear observer design consists of all systems (7) with outputs (8) that possess linearizable error dynamics. More precisely, these are systems $(7,8)$ that after a suitable state space transformation and output transformation can be brought in Lur'e form $(21,22)$. Conditions in terms of $f$ and $h$ that are necessary and sufficient for having linearizable error dynamics are given in (Nijmeijer and Mareels 1997) in the context of synchronization. As an illustration, consider the hyperchaotic Rössler system, see (Baier and Dahle 1995),

$$
\left\{\begin{aligned}
\dot{x}_{1} & =-x_{2}+a x_{1} \\
\dot{x}_{i} & =x_{i-1}-x_{i+1}, \quad i=2, \cdots, N-1 \\
\dot{x}_{N} & =\epsilon+b x_{N}\left(x_{N-1}-d\right)
\end{aligned}\right.
$$

with $a, b, d, \epsilon \in \mathbb{R}^{+}$and $N$ an arbitrary positive integer. The case $N=3$ corresponds to the usual Rössler system, and when $N=4$ the system has so-called hyperchaotic flows, and has two positive Lyapunov-exponents. With (26) we take as output equation

$$
y=x_{N}
$$

It is clear that the solutions of (26) with $x_{N}(0)>0$, and which exist for all positive time (no finite escape time!), have $x_{N}(t)>0$ for all $t>0$. Therefore we may introduce the coordinate transformation $z_{1}=x_{1}, \cdots, z_{N-1}=x_{N-1}, \dot{z}_{N}=\ell n\left(x_{N}\right)$, and output transformation $\bar{y}=\ell n(y)$. Then in the new coordinates the system reads as

$$
\begin{aligned}
\dot{z}_{1} & =-z_{2}+a z_{1} \\
\dot{z}_{i} & =z_{i-1}-z_{i+1}, \quad i=2, \cdots, N-2 \\
\dot{z}_{N-1} & =z_{N-2}-\exp \left(z_{N}\right) \\
\dot{z}_{N} & =b z_{N-1}-b d+\epsilon \exp \left(-z_{N}\right)
\end{aligned}
$$


and

$$
\bar{y}=z_{N}
$$

The remarkable fact is that the above system $(28,29)$ is again in Lur'e form $(21,22)$, and an easy check shows that the linear part is observable, thus allowing for a synchronizing system of the form (23).

The classes of systems for which a successful observer design is possible, sofar all exploit a linear error dynamics. There are however other cases where synchronization can be achieved without relying on a 'linearizability' assumption. To that end we return to the system (7) with measurement (8) and we introduce the following assumptions, see (Gauthier et al. 1992).

(i) The vector field $f$ in (7) satisfies a global Lipschitz condition on its domain, which as mentioned earlier need not to be $\mathbb{R}^{n}$.

(ii) The $n$ functions $h(x), L_{f} h(x), L_{f}^{2} h(x), \cdots, L_{f}^{n-1} h(x)$ define new coordinates (globally!). Here $L_{f}^{i} h(x)$ denotes the $i$-th iterated Lie-derivative of the function $h$ in the direction of $f$.

If both (i) and (ii) hold an observer exists of the form

$$
\dot{\tilde{x}}=f(\tilde{x})+K(h(x)-h(\tilde{x}))
$$

with $K$ a constant suitable $(n, 1)$-vector. Note that (30) obviously is of the form (14), though some of the entries in $K$ may become very large (high-gain). An illustrative example of a system that fulfills (i) and (ii) is formed by the Lorenz-system (1,2), when this is restricted to a compact domain. Since it is known that (1) has an attractive compact box, the observer (30) is an interesting alternative for the observer (3).

Besides the above discussed cases for which a synchronizing system can be systematically designed we note that there exist further methods that may be applicable for other classes of systems, like bilinear systems. Also for certain mechanical systems 'physics-based' observers can be developed, and finally some systems admit a Kalman filter-like observer. But, no general method exists that works for all systems.

\section{Uncertainty, robustness and noise}

In the previous section the synchronization problem has been treated under the assumption that the dynamics and output are exactly known. In many cases this is obviously not true and therefore alternative methods are required. We will review here three illustrative examples how one may possibly proceed in such case. The first example to be discussed contains parameter uncertainty in the dynamics ( 7$)$, i.e.

$$
\dot{x}=f(x, p)
$$

with $p$ some unknown parameter (vector), or, in a communications context, an unknown message. We take again as output

$$
y=h(x)
$$

Now, in addition to the standard synchronization problem of reconstructing $x(t), t \geq 0$, one may in addition be interested in reconstruction of the parameter $p$. The latter may be particularly interesting in a communications context where $p$ may represent some (slowly time-varying) signal. The next example illustrates that adaptive control (cf. (Sastry and Bodson 1989)) may form a good approach in such setting. Consider again the Chua circuit

$$
\left\{\begin{array}{l}
\dot{x}_{1}=\alpha\left(-x_{1}+y_{1}-\varphi\left(x_{1}, p\right)\right) \\
\dot{y}_{1}=x_{1}-y_{1}+z_{1} \\
\dot{z}_{1}=-\lambda y_{1}
\end{array}\right.
$$


where $\varphi\left(x_{1}, p\right)=\varphi\left(x_{1}\right)+p\left(\left|x_{1}+1\right|-\left|x_{1}-1\right|\right)=m_{1} x_{1}+\left(m_{2}+p\right)\left(\left|x_{1}+1\right|-\left|x_{1}-1\right|\right)$, with $m+1=\frac{5}{7}, m_{2}=-\frac{6}{7}, \lambda=14.286$ and $\alpha=9$. As output we take

$$
y=x_{1}
$$

The parameter $p$ is assumed to be constant or slowly time-varying, but in practice it may also be a binary time-varying signal. A solution to both the synchronization problem and the parameter estimation problem is given by the following adaptive observer

$$
\left\{\begin{array}{l}
\dot{x}_{2}=\alpha\left(-x_{2}+y_{2}-\varphi\left(x_{1}\right)\right)+\tilde{p}_{1}\left(\left|x_{1}+1\right|-\left|x_{1}-1\right|\right)+\tilde{p}_{2}\left(x_{2}-x_{1}\right) \\
\dot{y}_{2}=x_{2}-y_{2}+z_{2} \\
\dot{z}_{2}=-\lambda y_{2}
\end{array}\right.
$$

and

$$
\left\{\begin{array}{l}
\dot{\tilde{p}}_{1}=-\gamma_{1}\left(x_{1}-x_{2}\right)^{2} \\
\dot{\tilde{p}}_{2}=-\gamma_{2}\left(x_{1}-x_{2}\right)\left(\left|x_{1}+1\right|-\left|x_{1}-1\right|\right)
\end{array}\right.
$$

where $\gamma_{1}, \gamma_{2}>0$ are the positive adaptation gains. It follows, see (Fradkov et al. 1999), that again $\left(e_{1}, e_{2}, e_{3}\right)$ converges to $(0,0,0)$ but also $\tilde{p}_{1}$ and $\tilde{p}_{2}$ converge to their true values, and in particular $\tilde{p}_{1}$ converges to $p$, and $\tilde{p}_{2}$ can be viewed as an observer gain. The key observation in showing this result is actually the fact that the signal $\left|x_{1}+1\right|-\left|x_{1}-1\right|$ is 'persistently exciting' for the chaotic Chua circuit (33), which means this signal does not converge to some constant value. In case $p$ is a binary signal, the parameter convergence will occur provided the time step in changing $p$ is sufficiently large, see (Fradkov et al. 1999) for further details.

The idea of using adaptation mechanisms like in (36) requires that only parametric uncertainties occur. This may be a strong assumption in specific cases and alternatives may be sought. A simple illustration of a robust synchronization scheme can be given for a second order (mechanical) system

$$
\left\{\begin{array}{l}
\dot{x}_{1}=y_{1} \\
\dot{y}_{1}=f\left(x_{1}, y_{1}\right)
\end{array}\right.
$$

with

$$
y=x_{1}
$$

An observer is proposed as

$$
\left\{\begin{array}{l}
\dot{x}_{2}=y_{2}+k_{1}\left(x_{1}-x_{2}\right) \\
\dot{y}_{2}=k_{2}\left(x_{1}-x_{2}\right)
\end{array}\right.
$$

then, under the assumption that $f$ in (37) satisfies a global Lipschitz condition one may show that for $k_{1}, k_{2}>0$ sufficiently large, the error $\left(e_{1}, e_{2}\right)$ converges to a neighborhood of $(0,0)$ and moreover, the larger $k_{1}$ and $k_{2}$ are selected, the smaller the neighborhood of $(0,0)$ becomes. In this case we have a so-called high-gain observer (39) that achieves practical stability of the error $\left(e_{1}, e_{2}\right)$, and thus the state $\left(x_{2}, y_{2}\right)$ of (39) asymptotically almost synchronizes with $\left(x_{1}, y_{1}\right)$, see (Nijmeijer $1997 \mathrm{~b}$ ) for further details on the dual problem of robust control of chaotic systems. There exist in the control literature a wide range of alternative methods of studying robust observers, and thus robust synchronization; one alternative method can be found in (Pogromsky and Nijmeijer 1998), see also (Suykens et al. 1999). Besides parameter uncertainty or unstructured uncertainty in the dynamics (7) and output (8) the equations may be noisy. Noise may appear for different reasons in $(7,8)$, for instance measurement noise or uncertainties in the dynamics. In this case, synchronization becomes even more problematic than in the previous section, and certainly no exact state reconstruction will be possible. Nevertheless, a filtering approach may be very suited 
in this case and we will illustrate this through an example of a noisy Lorenz-system, see (Nijmeijer 1997a) for further details. Consider the Lorenz system with noise

$$
\left\{\begin{array}{l}
\dot{x}_{1}=\sigma\left(y_{1}-x_{1}\right)+\epsilon_{1} \\
\dot{y}_{1}=r x_{1}-y_{1}-x_{1} z_{1}+\epsilon_{2} \\
\dot{z}_{1}=-b z_{1}+x_{1} y_{1}+\epsilon_{3}
\end{array}\right.
$$

and noisy measurements

$$
y=x_{1}+v
$$

where $\left(\epsilon_{1}(t), \epsilon_{2}(t), \epsilon_{3}(t)\right) \sim N(0, Q)$ and $v(t) \sim N(0, R)$ are independent white noise processes. Clearly (40) now represents a set of stochastic differential equations, which due to the nonlinearities are highly nontrivial to solve (numerically). This makes it even more difficult to find a synchronizing system. Instead of a - deterministic - observer one may attempt to use an extended Kalman filter. (Here the word extended refers to the fact that the filter applies to nonlinear equations; the Kalman filter itself applies only to a linear stochastic system with noisy measurements.) The extended Kalman filter reads as

$$
\begin{cases}\dot{x}_{2}=\sigma\left(y_{2}-x_{2}\right) & +k_{1}(t) e_{1} \\ \dot{y}_{2}=r x_{2}-y_{2}-x_{2} z_{2} & +k_{2}(t) e_{1} \\ \dot{z}_{2}=-b z_{2}+x_{2} y_{2} & +k_{3}(t) e_{1}\end{cases}
$$

where, as before, $e_{1}=y-x_{2}=x_{1}+v-x_{2}$. At this point one may again notice that (42) fits in the structure (14). The crucial point of (42) lies in the way how the gain vector $k(t)=$ $\left(k_{1}(t), k_{2}(t), k_{3}(t)\right)^{T}$ is determined. For the filter $(42), k(t)$ is determined via

$$
k(t)=P(t)\left(\begin{array}{l}
1 \\
0 \\
0
\end{array}\right) R^{-1}
$$

where $R$ is the covariance of the measurement noise $v$ and $P(t)$ is the solution of the matrix Riccati differential equation

$$
\left\{\begin{aligned}
\dot{P} & =F(t) P+P F(t)^{T}-P H(t)^{T} R^{-1} H(t) P+Q \\
P(0) & =P_{0}>0
\end{aligned}\right.
$$

where $F(t)=\frac{\partial f}{\partial x}\left(x_{2}(t), y_{2}(t), z_{2}(t)\right)$ and $H(t)=\frac{\partial h}{\partial x}\left(x_{2}(t), y_{2}(t), z_{2}(t)\right)$ with $f$ and $h$ denoting the right hand side of (40) and (41). In other words $F(t)$ and $H(t)$ are obtained through linearization along the estimated solution $\left(x_{2}(t), y_{2}(t), z_{2}(t)\right)$. Although at this point no complete proof exists that guarantees that in some stochastic sense $\left(x_{2}(t), y_{2}(t), z_{2}(t)\right)$ converges approximately to $\left(x_{1}(t), y_{1}(t), z_{1}(t)\right)$, simulations indicate that with suitable initialization, the extended Kalman filter may form an appropriate scheme for synchronization, see (Sobiski and Thorp 1998). In the discrete-time context we have recently investigated this in detail for some specific chaotic systems, see (Cruz and Nijmeijer 2000).

Corollary 4 (i) Crucial in (Cruz and Nijmeijer 2000) is the observation that the chaotic systems under investigation 'live' in a compact region and thus fulfill a Lipschitz condition in this region. It is precisely this fact - which has some similarity with the high gain observer approach in the previous section - that enables a successful extended Kalman filter approach, see (LaScala et al. 1995).

(ii) It is clear that convergence of the estimate $\left(x_{2}(t), y_{2}(t), z_{2}(t)\right)$ towards $\left(x_{1}(t), y_{1}(t), z_{1}(t)\right)$ is at best possible in expectation. The noise in dynamics and measurement prohibit exact asymptotic convergence and therefore simulations based on the filter (42) will become sensitive with respect to the variances $Q$ and $R$. Likewise, the initialization of the Riccati differential equation (44) is an important design parameter. 


\section{Controlled synchronization}

Synchronization as reviewed in sections 2 and 3 was merely a property of finding an appropriate mechanism for reconstructing the state of some chaotic system on the basis of some given measurement signal. On the other hand this could be contrasted with another setting in which both transmitter and receiver dynamics are given, as well as the corresponding output function, and the aim is to find a suitable mechanism to control the slave system such that master and slave will asymptotically synchronize. More specifically, assume we have been given the transmitter

$$
\dot{x}=f(x)
$$

with output

together with the receiver dynamics

$$
y=h(x)
$$

$$
\dot{\bar{x}}=g(\tilde{x}, u)
$$

where we for simplicity assume that $x$ and $\tilde{x}$ both are $n$-dimensional. The dynamics (47) depends on a control (vector) $u$, which we assume for the moment to belong in $\mathbb{R}$. The control $u$ is the variable through which we may manipulate or change the dynamics (47), and it is here that we enter the area of nonlinear control, see (Nijmeijer and van der Schaft 1990). Obviously there exist many controller types that we may use but in the sequel we limit ourself to the use of a feedback of the form

$$
u=\alpha(\tilde{x}, y)
$$

where $\alpha$ is a smooth function depending on the state of the receiver and the available measurements of the transmitter. This is, at least at an intuitive level, a natural choice. The closed-loop system $(47,48)$ is now described as

$$
\dot{\tilde{x}}=g(\tilde{x}, \alpha(\tilde{x}, h(x))
$$

and the aim in synchronizing master and slave system now is to find a suitable function $\alpha$ in (48) such that asymptotically $x(t)$ and $\tilde{x}(t)$ coincide. Stated in terms of section 2 this implies that (49) acts as an observer for (45).

Corollary 5 iFrom the foregoing it becomes clear that there are numerous other ways to enforce the synchronization between (45) and (46). In (Blekhman et al. 1997) various definitions are given, with perhaps the most general controller being of the form

$$
\left\{\begin{array}{l}
u=\alpha(\tilde{x}, z, y) \\
\dot{z}=h(z, y)
\end{array}\right.
$$

which is in control terminology a dynamic output feedback. Potentially the introduction of the dynamics in (50) allows for synchronization of (45) and (47,50), which means that in this case we need not to start with systems (45) and (46) of the same dimension.

The general problem of finding, if possible, a suitable output feedback (48) in order that (45) and (49) synchronize is quite difficult. We will illustrate this by means of a relatively simple example of van der Pol systems. Consider as transmitter dynamics the van der Pol system

$$
\left\{\begin{array}{c}
\dot{x}_{1}=y_{1} \\
\dot{y}_{1}=-x_{1}-\left(x_{1}^{2}-1\right) y_{1} \\
y=x_{1}
\end{array}\right.
$$

and as receiver we take the 'controlled' van der Pol system

$$
\begin{cases}\dot{x}_{2}=y_{2} & +\alpha u \\ \dot{y}_{2}=-x_{1}-\left(x_{1}^{2}-1\right) y_{2} & +\beta u\end{cases}
$$


Note that we have exploited the knowledge of $x_{1}$ in (53), and also that control in (53) is possible along the direction $(\alpha \beta)^{T}$. If (53) represents an electrical circuit or physical system it may happen that either $\alpha=0$ or $\beta=0$. Typically the control $u$ is a current (or voltage) or force that acts on the system.

Corollary 6 At this point, there is a notable difference with most of the 'control of chaos' literature where often a control parameter is varied as to influence the dynamics, see for instance the OGY paper (Ott et al. 1990).

To achieve synchronization of (52) and (53) we will use here (high-gain) output error feedback $\left(c_{1}>0\right)$

$$
u=-c_{1}\left(x_{1}-x_{2}\right)
$$

resulting in the error dynamics

$$
\left\{\begin{array}{l}
\dot{e}_{1}=-\alpha c e_{1}+e_{2} \\
\dot{e}_{2}=-\beta c e_{1}-\left(x_{1}^{2}-1\right) e_{2}
\end{array}\right.
$$

which is a linear time-varying system. For the synchronization of $(51)$ and $(53,54)$ it is required that the error dynamics (55) are asymptotically stable about the equilibrium $(0,0)$. Already this relatively simple error dynamics require some nontrivial analysis. The most interesting case probably arises if $\alpha=0$ and $\beta \neq 0$. One can show, see (Huijberts et al. 2000b), that there exists a constant $c_{*}$ - which is determined in terms of the transmitter dynamics (51) - such that if the gain $c>c_{*}$, then the error dynamics (55) are uniformly exponentially stable. In fact, this result follows by transforming (55) to an associated Hill-equation and as a result the stability turns out to be rather slow. At the same time, the lower bound form (Huijberts et al. 2000b) may be rather conservative.

The example of controlled synchronization reveals that the problem to find a suitable (output) feedback controller that achieves synchronization of transmitter and receiver will in general become difficult, or even impossible to solve. On the other hand, a systematic analysis that parallels the different cases reviewed in section 2 may lead to other solutions. For instance, this is true for Lur'e systems, with a transmitter system of the form

$$
\begin{gathered}
\dot{x}=A x+\varphi(C x) \\
y=C x
\end{gathered}
$$

and receiver dynamics

$$
\dot{\tilde{x}}=A \tilde{x}+\varphi(C x)+B u
$$

It follows that provided the pair $(A, C)$ is detectable, as well as $\left(A^{T}, B^{T}\right)$ is detectable (or equivalently, $(A, B)$ is stabilizable) then there exists a (linear) dynamic output feedback of the form (50) such that the two systems asymptotically synchronize. For further details and insight in the controlled synchronization problem the reader is referred to (Huijberts et al. 2000b).

\section{Epilogue}

We have tried to give a dynamical control view on synchronization. All in all, it is felt that nonlinear control may provide some useful tools to address certain synchronization problems. On the other hand, in many cases, a thorough study of certain time-varying dynamical systems is required and it may be concluded that further research along these lines requires knowledge from both dynamical systems and nonlinear control theory. The review as presented here gives only a partial view on synchronization. There are numerous variants of synchronization defined in the literature, of which one could mention, phase synchronization, partial synchronization and generalized synchronization, see (Parlitz and Kocarev 1999), or (Blekhman et al. 1997) where 
a general definition of (controlled) synchronization is proposed. In the study of synchronization several elements from control theory turn out to be relevant. This includes observers (see section 2 ), filtering and robustness (section 3) and feedback control (section 4), but also further aspects as system inversion, cf. (Feldman at al. 1996) or system identification, cf (Huijberts et al. 2000). It should be clear that synchronization problems can be treated in other domains too. In particular, for discrete-time systems various results more or less parallel the material from the foregoing sections. Even for transmitter/receiver dynamics described by partial differential equations one may expect some results along these lines, see e.g. (Chen 1999) for a specific example of synchronizing pdes. Likewise, synchronization with time-delayed feedback has also been studied in (Chen 1999). Synchronization has numerous potential applications running from coordination problems in robotics to mechanisms for secure communications. Precisely the latter area was mentioned in (Pecora and Carroll 1990) as a potential field of application, although sofar much work remains to be done here.

\section{References}

[1] Baier, G. \& Sahle, S. 1995 Design of hyperchaotic flows. Physical Review E, 51, no. 4, R27122714.

[2] Blekhman, I. I., Landa, P. S. \& Rosenblum, M. G. 1995 Synchronization and chaotization in interacting dynamical systems. Appl. Mech. Rev. 48, no. 11, 733-752.

[3] Blekhman, I. I., Fradkov, A. L., Nijmeijer, H. \& Pogromsky, A.Yu. 1997 On selfsynchronization and controlled synchronization. Syst. Control Letters, 31, 299-305.

[4] Brunt, M. 1998 Coordination of redundant systems, PhD-dissertation, Technical University Delft, The Netherlands.

[5] Chen, G. (ed.) 1999 Controlling chaos and bifurcations in engineering systems, CRC Press, Boca Raton.

[6] Corron, N. J. \& Hahs, D. 1997 A new approach to communications using chaotic signals. IEEE Trans. Circuits and Syst. I, 44, 373-382.

[7] Cruz, C. \& Nijmeijer, H. (to appear) March 2000 Synchronization through filtering. Int. Jnl. of Bifurcation and Chaos, Theme issue "Control and Synchronization of chaotic systems."

[8] Feldman, U. Hasler, M. \& Schwarz, W. 1996 Communication by chaotic signals: the inverse system approach. Int. Inl. Circ. Theory Applic., 24, 551-579.

[9] Fradkov, A. L., Nijmeijer, H. \& Pogromsky, A.Yu. 1999 Adaptive observer based synchronization, In: (Chen 1999), 417-435.

[10] Gauthier, J. Hammouri, H. \& Othman, S 1992 A simple observer for nonlinear systems, applications to bioreactors. IEEE Trans. Automatic Contr. 37, 875-880.

[11] Huijberts, H. J. C., Nijmeijer, H. \& Willems, R. (to appear) 2000a System identification in communication with chaotic systems. IEEE Trans. Circuits and Systems I.

[12] Huijberts, H. J. C., Nijmeijer, H. \& Willems, R. (to appear) 2000b Regulation and controlled synchronization for complex dynamical systems. Int. Jnl. Robust and Nonl. Contr.

[13] Huijgens, C. 1673 Horologium Oscilatorium, Paris, France.

[14] Fradkov, A. L., Nijmeijer, H. \& Pogromsky, A. Yu. 1999 Adaptive observer based synchronization. in (Chen, 1999), 417-438.

[15] Kailath, T. 1980 Linear Systems, Englewood Cliffs, NJ: Prentice-Hall. 
[16] LaScala, B. F., Bitmead, R. R. \& James M. R. 1995 Conditions for stability of the extended Kalman filter and their application to the frequency tracking problem. Mathem. Control, Signals and Syst., 8, 1-26.

[17] Liu, Y.-H., Xu, Y. \& Bergerman, M. 1999 Cooperation control of multiple manipulators with passive joints. IEEE Trans. Robotics and Automation, 15, 258-26-'.

[18] Nijmeijer, H. \& Mareels, I. M. Y. 1997 An observer looks at synchronization. IEEE Trans. Circuits and Systems I, 44, 882-890.

[19] Nijmeijer, H. 1997a On synchronization of chaotic systems. Proceedings 36th IEEE Conference on Decision and Control, San Diego, 384-388.

[20] Nijmeijer, H. 1997b Adaptive/robust control of chaotic systems. Proc. IUTAM Symp. on Interaction between Dynamics and Control in Advanced Mechanical Systems, van Campen, D. H. (ed.), Kluwer Academic Publishers, 255-262.

[21] Nijmeijer, H. \& van der Schaft, A. J. 1990 Nonlinear Dynamical Control Systems, Springer, New York.

[22] Ott, E., Grebogi, C. \& Yorke, J. A. 1990 Controlling chaos. Phys. Review Letters, 64, 11961199.

[23] Parlitz, U. \& Kocarev, L. 1999 Synchronization of chaotic systems, in Schuster, H. G. (ed.), Handbook of chaos control, Wiley-VCH, New York, 272-302.

[24] Pecora, L. M. \& Carroll, T. L. 1990 Synchronization in chaotic systems. Phys. Review Lett., 64, 821-824.

[25] Pol van der, B. 1920 Theory of the amplitude of free and forced triod vibration. Radio Rev., $1,701-710$.

[26] Pogromsky, A. Yu. \& Nijmeijer, H.1998 Observer based robust synchronization of dynamical systems. Int. Jnl. Bifurcation and Chaos, 8, 2243-2254.

[27] Pyragas, K. 1992 Continuous control of chaos by self-controlling systems. Phys. Lett. A, 170, $421-428$

[28] Rayleigh, J. 1945 Theory of sound, Dover Publ.

[29] Sastry, S. \& Bodson, M. 1989 Adaptive control-stability, convergence and robustness, Prentice Hall, Englewood Cliffs.

[30] Sobiski, D. J. \& Thorp, J. S. 1998 PDMA-1 Chaotic communication via the extended Kalman filter. IEEE Trans. Circuits and Systems I, 45, 194-197.

[31] Suykens, J. A. K., Curran, P. F. \& Chua, L. O. 1999 Robust synthesis for master-slave synchronization of Lur'e systems. IEEE Trans. Circuits and Systems I, 46, 841-850. 